\title{
Current sustainability and electromigration of Pd, Sc and $Y$ thin-films as potential interconnects
}

\author{
Yong Yang, Shengyong Xu*, Sishen Xie and Lian-Mao Peng*
}

The progress on novel interconnects for carbon nanotube (CNT)-based electronic circuit is by far behind the remarkable development of $\mathrm{CNT}$-field effect transistors. The $\mathrm{Cu}$ interconnect material used in current integrated circuits seems not applicable for the novel interconnects, as it requires electrochemical deposition followed by chemical-mechanical polishing. We report our experimental results on the failure current density, resistivity, electromigration effect and failure mechanism of patterned stripes of $\mathrm{Pd}, \mathrm{Sc}$ and $\mathrm{Y}$ thin-films, regarding them as the potential novel interconnects. The Pd stripes have a failure current density of $(8 \sim 10) \times 10^{6} \mathrm{~A} / \mathrm{cm}^{2}\left(\mathrm{MA} / \mathrm{cm}^{2}\right)$, and they are stable when the working current density is as much as $90 \%$ of the failure current density. However, they show a resistivity around $210 \mu \Omega \cdot \mathrm{cm}$, which is 20 times of the bulk value and leaving room for improvement. Compared to Pd, the Sc stripes have a similar resistivity but smaller failure current density of 4 5 $\mathrm{MA} / \mathrm{cm}^{2}$. Y stripes seem not suitable for interconnects by showing even lower failure current density than that of Sc and evidence of oxidation. For comparison, Au stripes of the same dimensions show a failure current density of $30 \mathrm{MA} / \mathrm{cm}^{2}$ and a resistivity around $4 \mu \Omega \cdot \mathrm{cm}$, making them also a good material as novel interconnects.

Keywords: Carbon nanotube-based field effect transistors; Carbon nanotube-based circuit; Interconnects; Current density; Electromigration; Resistivity

Citation: Yong Yang, Shengyong Xu, Sishen Xie, and Lian-Mao Peng, "Current sustainability and electromigration of Pd, Sc and Y thin-films as potential interconnects", Nano-Micro Lett. 2, 184-189 (2010). doi: 10.5101/nml.v2i3.p184-189

To construct novel carbon nanotube (CNT)-based nanoelectronic circuits that follow the well-developed models of Si-based CMOS technology, one needs both $p$-type and $n$-type CNT-based field effect transistors (CNT-FETs), and proper interconnects. The equally high mobility of both electrons and holes in CNTs make it possible to fabricate high-performance $p$-type and $n$-type CNT-FETs with symmetric device parameters which are superior to those of conventional Si-based FETs [1-4]. However, progress on novel interconnects for the CNT-based electronic circuit is by far behind the remarkable development of CNT-FETs. In CNT-based electronics circuits, the key element of each individual device is a short piece of semiconducting CNT with diameter of only $1 \sim 2 \mathrm{~nm}$. Hence the interconnect material used in current integrated circuits, $\mathrm{Cu}$, seems not applicable, as it requires electrochemical deposition of $\mathrm{Cu}$ layers followed by chemical-mechanical polishing [5], which may easily damage the fragile structures of CNT-based devices.

Theoretically it is predicted that multi-walled CNT (MWCNT) could be a good candidate as interconnect for novel nanoelectronics [6-8]. Because MWCNTs are chemically and thermally stable and can sustain an extremely high working current density. But in practice, conventional lithography and thin-film technologies are not applicable for the MWCNTs, and it is hard to manipulate and/or grow MWCNTs with desired length and diameter to the target positions in a circuit, therefore to date only a few demonstrations have been reported that MWCNTs can be used as part of the interconnects in a prototype electronic circuit [9-11]. 
Since the state-of-the-art $p$-type CNT-FETs require palladium (Pd) electrodes [1], and $n$-type CNT-FETs require scandium $(\mathrm{Sc})$ or yttrium $(\mathrm{Y})$ electrodes $[2,4]$, it is a natural choice to use the same electrode materials as the interconnects for the novel CNT-FET-based circuits, if any of them fulfills the requirements of such interconnects. This allows the fabrication of electrodes (either for $p$-type or $n$-type FETs) and interconnects in one deposition step thus simplifying the processing, and also maximally reduces the risk of contamination and interface effect that a metal other than the electrode materials (i.e., Pd, Sc or Y) brings to the circuit, which may degrade the performance of CNT-FETs.

For an interconnect material, sustainability of current density, resistivity, chemical and thermal stability, as well as technical compatibility and cost are some of the key parameters to be considered. Electromigration, which may lead to opens or shorts in interconnects of an integrated circuit after hours of use, is another important factor [12-19]. Indeed, the change of interconnects from $\mathrm{Al}$ to $\mathrm{Cu}$ in the development of integrated circuit industry is partly because of the electromigration [20-22]. Here we report our experimental results on the current sustainability, resistivity and electromigration effects of patterned stripes of $\mathrm{Pd}, \mathrm{Sc}$ and $\mathrm{Y}$ thin-films, as well as Au stripes for a comparison, regarding them as potential interconnects.

Thin-film stripes with thickness of $40 \sim 80 \mathrm{~nm}$, width of $1 \sim 4$
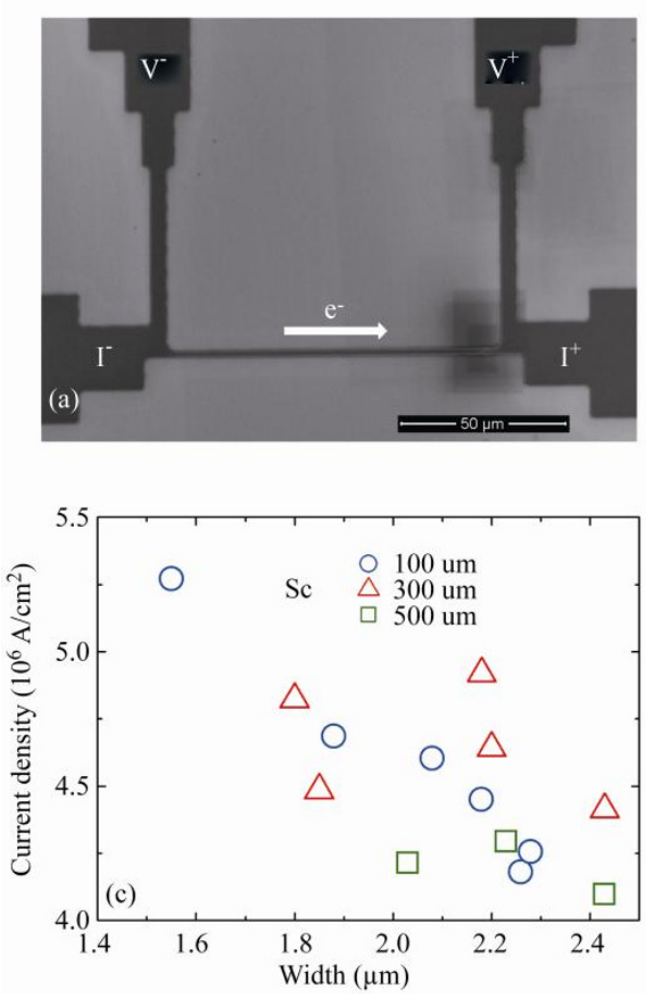

$\mu \mathrm{m}$ and length of $100,300,500,700$ and $1000 \mu \mathrm{m}$ are made into a 4-lead structure (as shown in Fig. 1a) on $\mathrm{SiO}_{2}(500 \mathrm{~nm}) / \mathrm{Si}$ substrates by using standard photolithography techniques. The $\mathrm{Pd}, \mathrm{Sc}$ and $\mathrm{Y}$ thin films are fabricated with an electron-beam deposition system (Kurt J. Lesker, AXXIS), which has a base vacuum of $5 \times 10^{-9}$ torr. The $\mathrm{Au}$ films are made with a sputtering system (Kurt J. Lesker, KJLC PVD 75), which has a base vacuum of $8 \times 10^{-8}$ torr. Film thickness is measured by using an Ambios XP-1 Stylus Profiler. The crystalline structure of the films is measured with an X-ray diffraction system (SHIMADZU, XRD-6000). The resistance of the samples is measured with a home-made automation system consists of source meters Keithley 2400 and Keithley 2410, which has a measurement error around $0.05 \%$ for 4-lead resistivity measurements. The failure current density values of $\mathrm{Pd}, \mathrm{Sc}, \mathrm{Y}$ and $\mathrm{Au}$ stripes are measured first from 2 3 samples of each materials, then a working current with current density set at approximately $60 \%, 70 \%, 80 \%, 90 \%$ and $100 \%$ of the measured failure current density is applied to a fresh sample, respectively, each for 40 hours (unless the sample breaks shortly).

The interconnection for novel CNT-based integrated circuit may be required to have a limited line-width and thickness (thus a small cross-sectional area), but to support a working current up to $\mathrm{mA}$. As a result, they should sustain a current density up to $\mathrm{MA} / \mathrm{cm}^{2}$. From the Pd stripe samples (see Fig.1a) with varied
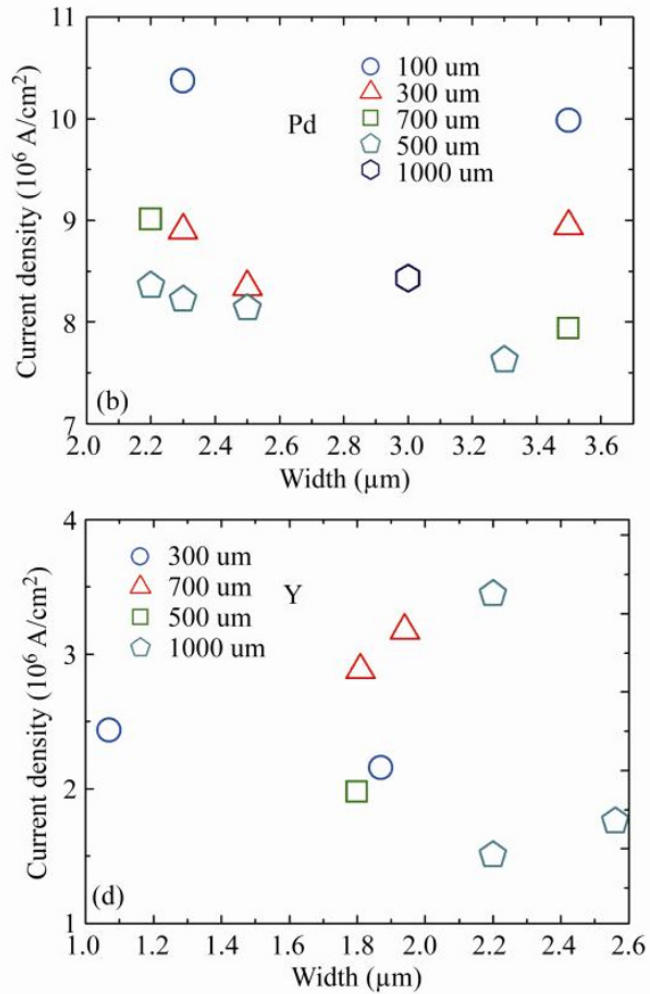

FIG. 1. (a) SEM image of a $100 \mu \mathrm{m}$ long stripe sample. The arrow shows the motion direction of electrons under test; (b) Plot of the failure current densities of Pd stripes; (c) Failure current densities of Sc stripes; (d) Failure current densities of Y stripes. 
width from 2.2 to $3.5 \mu \mathrm{m}$, failure current densities (at which the stripe breaks) of 7.6 10.4 MA/ $\mathrm{cm}^{2}$ are measured, as shown in Fig. 1b. Thus for a Pd stripe of $1 \mu \mathrm{m}$ wide and $50 \mathrm{~nm}$ thick, for instance, it can sustain a maximum current of $4 \sim 5 \mathrm{~mA}$. The failure current densities in Sc stripes and Y stripes are mostly $4 \sim 5 \mathrm{MA} / \mathrm{cm}^{2}$ and 1.5 3.5 MA/cm ${ }^{2}$, respectively (see Fig. 1c and Fig. 1d). Though the values are sample dependent, clearly Pd stripes can sustain much higher working current density than Sc and Y stripes do.

The 4-lead structure of the stripe (see Fig. 1a) enables us to obtain the resistivity values of these samples directly. The average resistivity values of $\mathrm{Pd}, \mathrm{Sc}$ and $\mathrm{Y}$ stripes are measured to be around 216,213 , and $265 \mu \Omega \cdot \mathrm{cm}$, respectively. Surprisingly, the measured resistivity of our Pd thin films is about 20 times of the Pd bulk resistivity [23]. In sharp contrast, the resistivity values for Sc and Y stripes are only 3.5 and 4.5 times of the bulk values of their counterparts, respectively. This implies a big room for improving the quality of Pd film, though the present $\mathrm{Pd}$ film has already shown a high current sustainability.

We have studied the electromigration effects of the samples by monitoring the change of resistance when a large working current is running in a stripe for a long period of time up to 200 hours. For Pd stripes, as typically shown in Fig. 2a, an obvious increase of resistance with time can be observed under a working current of $5.12 \mathrm{MA} / \mathrm{cm}^{2}$, which is $75 \%$ of the failure current
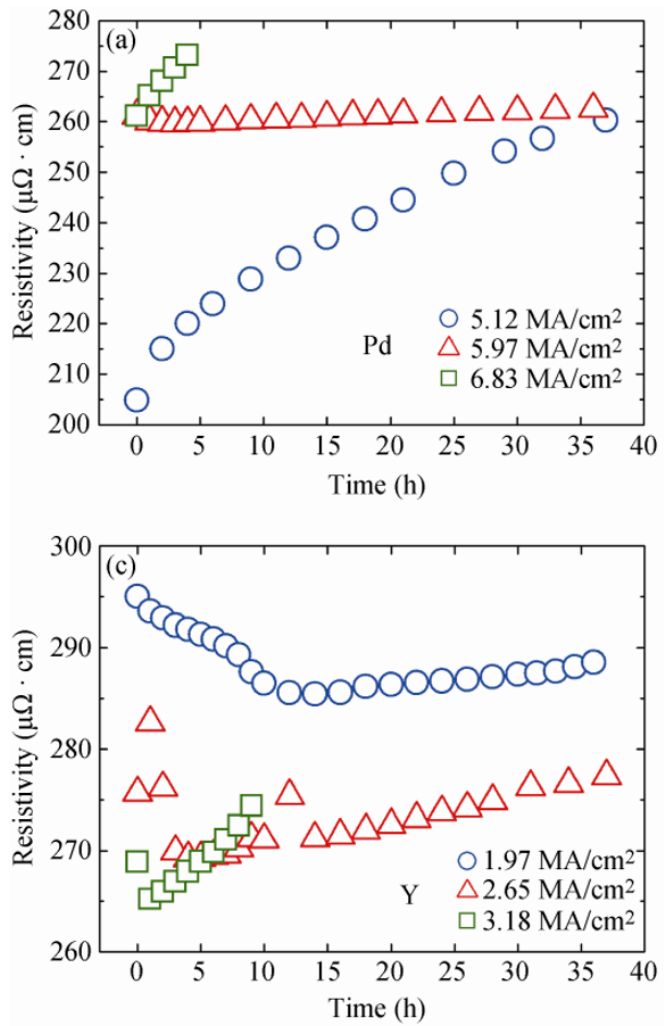

density $\sim 6.83 \mathrm{MA} / \mathrm{cm}^{2}$. Next, by increasing the current density to $5.97 \mathrm{MA} / \mathrm{cm}^{2}$, and about $87 \%$ of the failure current density, the sample keeps in a steady regime by showing a slowly increasing resistance. Under a working current of $6.83 \mathrm{MA} / \mathrm{cm}^{2}$, it causes a quick increase of resistance until the stripe breaks. For Sc stripes, typically the sample resistance shows a decrease under a working current of $50 \sim 60 \%$ of the failure current density, then a quick increase when the working current keeps on increasing (see Fig. 2b), until the stripe breaks. Y stripes, however, show a random trend. Figure $2 \mathrm{c}$ plots the measured data of one of the $\mathrm{Y}$ samples. The phenomenon of linear increasing resistance with time, similar to what is measured in our Pd stripes, has been observed previously in $\mathrm{Cu}$ and $\mathrm{Al}(4 \% \mathrm{Cu})[17,24]$. Theoretically this is attributed to the dynamics of vacancy and defects in the electromigration process [15,25-27]. On the other hand, the annihilation of vacancy and imperfection can lead to decrease of the resistance at the beginning of the electromigration in low current [15], as what is observed in our Sc stripes.

When running a current close to the maximum sustainable value (i.e., the failure current) in a stripe for a long time, or when the current density exceeds the maximum sustainable value, it results in break of the stripe thus failure of the circuit. Three underlying mechanisms that may result in break (failure) of a thin-film stripe are electromigration-induced stress, thermomechanical stress, and heat-induced melting effect
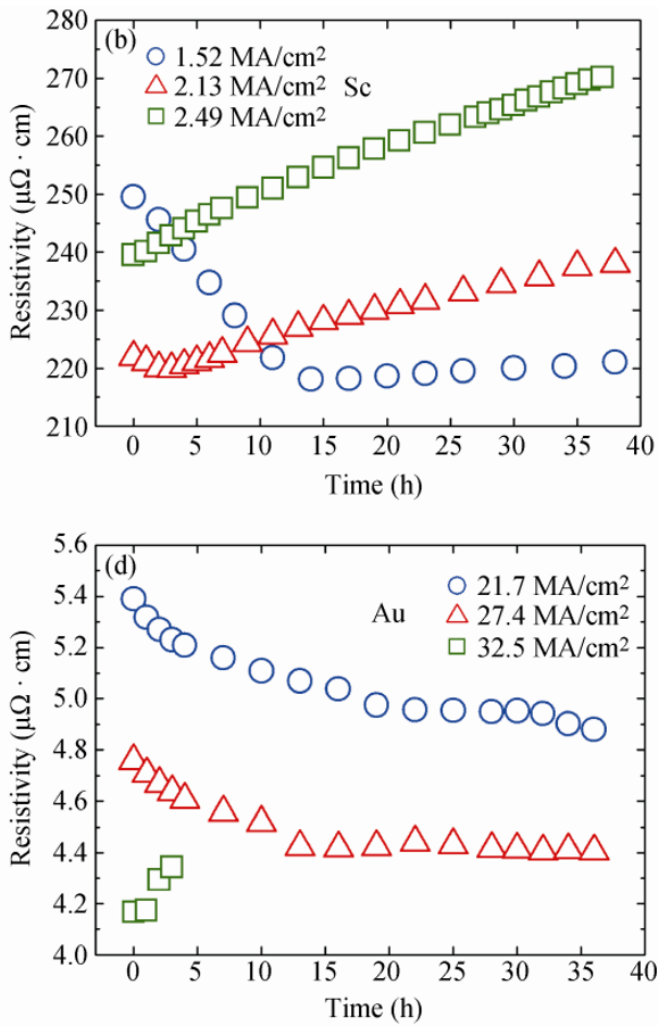

FIG. 2. (a)-(d) Resistivity versus time under increasing working current in Pd, Sc, Y and Au stripes, respectively. The environment temperature is kept at $300 \mathrm{~K}$. 
[28-31]. It is predicted that when the current is not high enough to induce significant Joule heating, the stress is tensile at the cathode end and compressive at the anode end [28]. Because materials fracture more easily under tensile stress than under compressive stress, a thin film is expected to fail at the cathode under low current. As the current level increases and Joule heating becomes more dominant, the whole stress (electromigration and thermomechanical) turns to being compressive, so the film will fail at the anode end. In the third situation, the current is large enough so that the electromigration stress is smaller than the thermonechanical stress, then the film is expected to fail catastrophically toward the center.

Figure 3 shows scanning electron microscope images of a Pd stripe before and after it breaks. The break region is melting-like, as shown in Fig. 3c and Fig. 3d, which might be caused by the combination of electromigration force and Joule heating effect. Figure 4 shows that, for the same Pd stripe, after the stripe is broken by running a high working current for a long time, the change of surface morphology is obvious at the anode region (see Fig. 4c and Fig. 4d), but not at the cathode region (see Fig. 4a and Fig. 4b). This is consistent with the theoretical predictions [28].

Y films can wet well to CNTs and make Ohmic contact [32]. However, it can also be easily oxidized, and indeed this nature has been applied to obtain high-performance gate dielectrics for graphene-based devices [33]. Weak peaks of $\mathrm{Y}_{2} \mathrm{SiO}_{5}$ (or a mixture of $\mathrm{Y}_{2} \mathrm{O}_{3}$ and $\mathrm{SiO}_{2}$ ) are found in the X-ray diffraction patterns of the $\mathrm{Y}$ films. Formation of oxides is surely not favorable for the materials to be used as interconnects. At the break points of Y stripes, often plenty of voids are observed (see Supplementary).
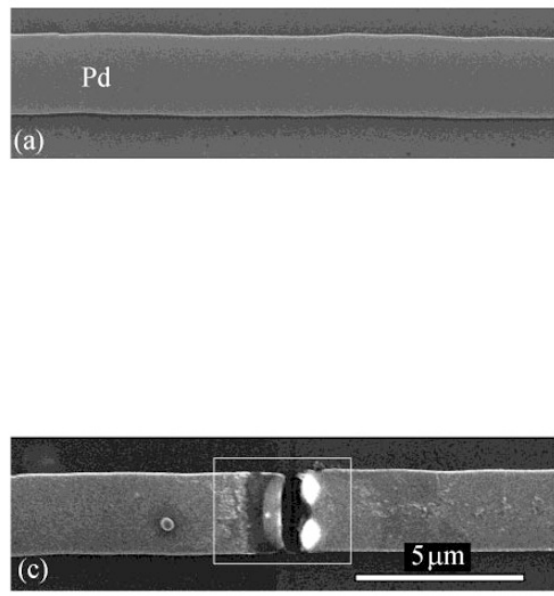

For a comparison, we have also measured the similar properties of Au stripes. An average failure current density of 30 $\mathrm{MA} / \mathrm{cm}^{2}$ is measured, which is slightly higher than $29 \mathrm{MA} / \mathrm{cm}^{2}$ as reported by Hadeed and Durkan [28]. The resistivity of $\mathrm{Au}$ stripes is measured to be $4.0 \sim 4.3 \mu \Omega \cdot \mathrm{cm}, 1.8$ times of the bulk resistivity of $\mathrm{Au}, 2.35 \mu \Omega \cdot \mathrm{cm}$. As typically shown in Fig. $2 \mathrm{~d}$, the resistance of an Au stripe keeps on decreasing in a low working current. When the working current is approaching the maximum sustainable value, the resistance starts to increase quickly till break of the stripe. This behavior is different from those observed in Pd and Sc stripes. After the Au stripes are broken, big particles and voids are observed in the samples (see Supplementary).

In conclusion, $\mathrm{Pd}$ is a potential candidate as the interconnect material for novel CNT-FET-based circuits. A failure current density of $8 \sim 10 \mathrm{MA} / \mathrm{cm}^{2}$ is measured in the Pd micro-stripes, and they are remarkably stable when the working current density is as much as $80 \sim 90 \%$ of the maximum sustainable density. However, our Pd samples show a resistivity around $210 \mu \Omega \cdot \mathrm{cm}$, which is 20 times of the bulk value, leaving plenty of room for further improvement. The Sc stripes show similar resistivity but half of the failure current density compared to those of $\mathrm{Pd}$ samples. Y films seem not suitable for interconnects when they show much lower failure current density than Pd and Sc, and evidence of oxidation. For comparison, Au films could also be considered as a good interconnect material, which has a failure current density of $30 \mathrm{MA} / \mathrm{cm}^{2}$ and a resistivity around $4 \mu \Omega \cdot \mathrm{cm}$, providing that the diffusion of $\mathrm{Au}$ atoms dose not degrade the performance of novel CNT-FETs.

We thank W. Q. Sun and Z. X. Wang for valuable discussions.
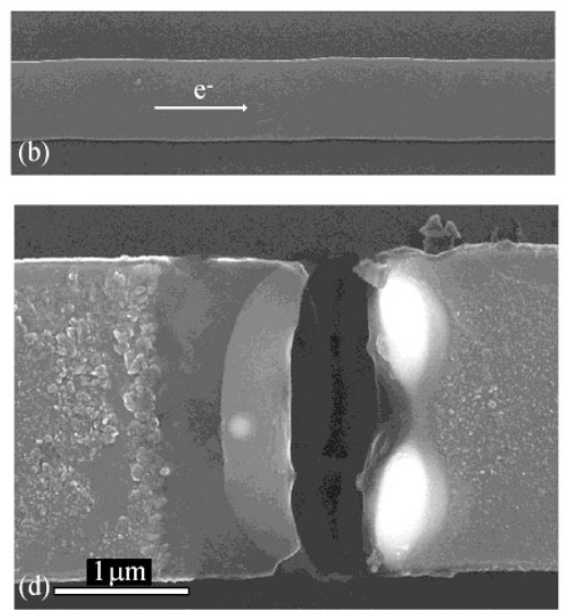

FIG. 3. (a)-(d) SEM images of the same Pd stripe (2.42 $\mu \mathrm{m}$ wide, $300 \mu \mathrm{m}$ long) before and after it has been running a working current for long time. (a) original morphology; (b) after running a current of $11 \mathrm{~mA}$ (corresponding to a current density of $5.93 \mathrm{MA} / \mathrm{cm}^{2}$ ) for 4 hours; (c) after subsequently running a working current of 15 $\mathrm{mA}$ (corresponding to a current density of $8.09 \mathrm{MA} / \mathrm{cm}^{2}$ ) for 1 more hour, causing break at the center of the stripe; (d) A close look at the broken region as highlighted in (c), showing melting-like morphology. 

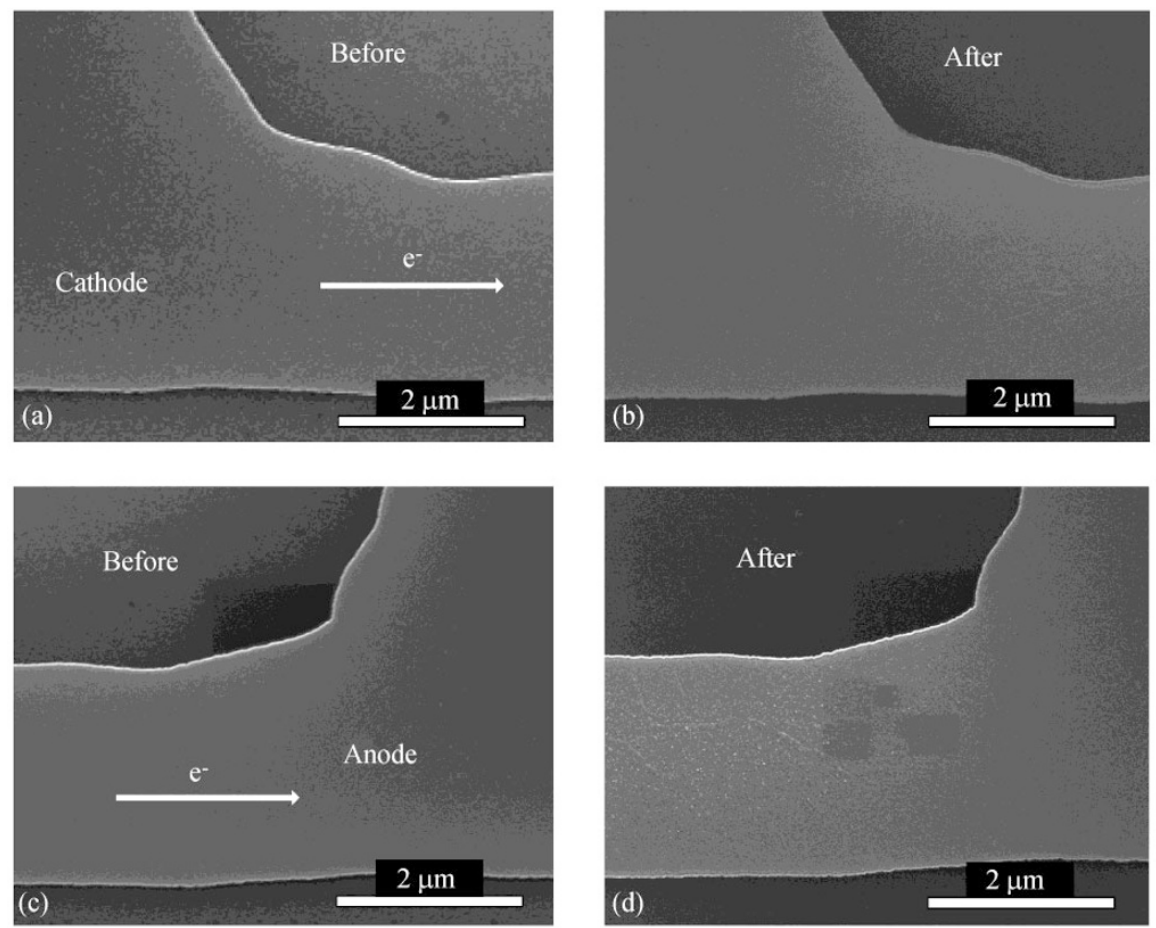

FIG. 4. The surface morphology change of a Pd stripe $(2.42 \mu \mathrm{m}$ wide, $300 \mu \mathrm{m}$ long) before and after it is broken at the central region after running a working current of density of $5.93 \mathrm{MA} / \mathrm{cm}^{2}$ for 4 hours then $8.09 \mathrm{MA} / \mathrm{cm}^{2}$ for 1 hour. (a)- (b) The same Pd strip at the cathode region. (c)- (d) The same Pd strip at the anode region.
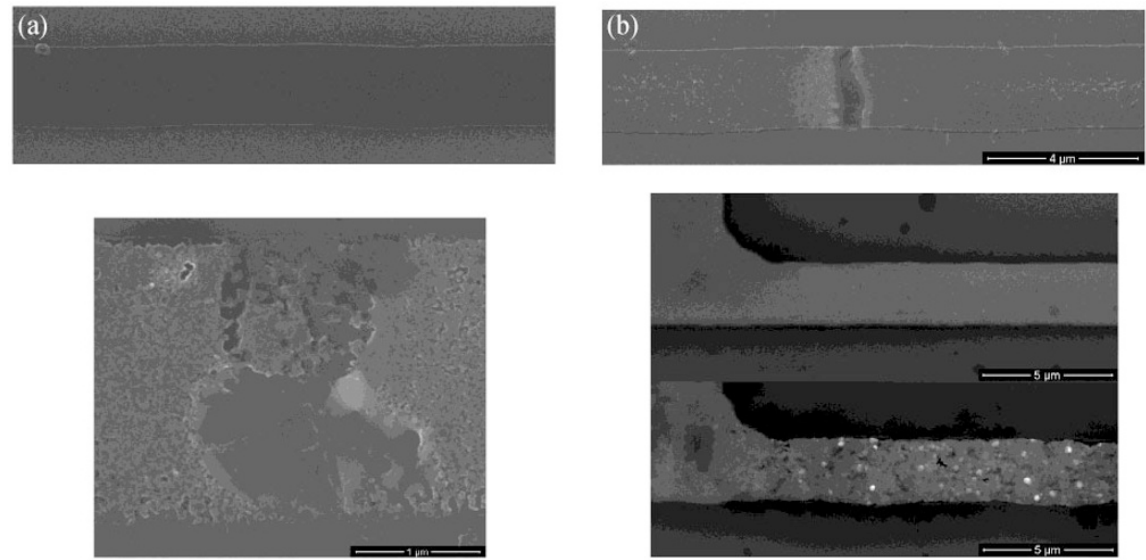

SUPPLEMENTARY Morphology changes in Sc (a \& b), Y (c), and Au (d \& e) stripes before and after failure of the sample. Electromigration induced voids can clearly be seen in Y (c) and Au stripes (d \& e) after the failure occurs. For the Sc stripe (a \& b), it has been running a working current of $9.3 \mathrm{~mA}$ (corresponding to a current density of $5.47 \mathrm{MA} / \mathrm{cm}^{2}$ ) for 5 hours. For the Au stripe (d \& e), it has been running a working current of $31.5 \mathrm{~mA}$ (corresponding to a current density of $30.8 \mathrm{MA} / \mathrm{cm}^{2}$ ) for 0.3 hours.

This work was supported by the NSF China (10774002) and the MOST China (No 2006CB932401).

\section{Received 19 August 2010; accepted 16 September 2010; published online 29 September 2010.}

\section{References}

1. A. Javey, J. Guo, Q. Wang, M. Lundstrom and H. J. Dai, Nature. 424, 654 (2003). doi:10.1038/nature01797
2. Z. Y. Zhang, X. L. Liang, S. Wang, K. Yao, Y. F. Hu, Y. Z. Zhu, Q. Chen, W. W. Zhou, Y. G. Yao, J. Zhang and L.M. Peng, Nano Lett. 7, 3603 (2007). doi:10.1021/n10717107

3. Z. Y. Zhang, S. Wang, L. Ding, X. L. Liang, H. L. Xu, J. Shen, Q. Chen, R. L. Cui, Y. Li and L.M. Peng, Appl. Phys. Lett. 92, 133117 (2008). doi:10.1063/1.2907696

4. L. Ding, S. Wang, Z. Y. Zhang, Q. S. Zeng, Z. X. Wang, T. Pei, L. J. Yang, X. L. Liang, J. Shen, Q. Chen, R. L. Cui, Y. Li and L.M. Peng, Nano Lett. 9, 4209 (2009). $\underline{\text { doi:10.1021/n19024243 }}$ 
5. R. Doering and Y. Nishi, Handbook of semiconductor manufacturing technology, CRC Press, Talyor \& Francis Group, Boca Raton, (2008).

6. P. J. Burke, IEEE Trans. Nanotechnolology 1, 129 (2002). doi:10.1109/TNANO.2002.806823

7. M. J. Hagmann, IEEE T. Nanotechnol. 4, 289 (2005). doi:10.1109/TNANO.2004.842040

8. A. Raychowdhury and K. Roy, IEEE T. Comp.-Aided Design Integ. Circ. Sys. 25, 58 (2006). doi:10.1109/TCAD. $\underline{2005.853702}$

9. G. F. Close, S. Yasuda, B. Paul, S. Fujita and H.S. Philip Wong, Nano Lett. 8, 706 (2008). doi:10.1021/n10730965

10. X. L. Liang, S. Wang, X. L. Wei, L. Ding, Y. Z. Zhu, Z. Y. Zhang, Q. Chen, Y. Li, J. Zhang and L.M. Peng, Adv. Mater. 21, 1339 (2009). doi:10.1002/adma.200802758

11. M. S. Wang, J. Y. Wang, Q. Chen and L.M. Peng, Adv. Funct. Mater. 15, 18 (2005).

12. L. Baozhen, D. S. Timothy, C. L. Tom and B. Dinesh, Microelectron. Reliab. 44, 365 (2004). doi:10.1016/j. microrel.2003.11.004

13. C. K. Hu, L. M. Gignac, E. Liniger, E. Huang, S. Greco, P. McLaughlin, C. C. Yang and J. J. Demarest, Stress-induced phenomena in metallization, AIP Conf. Proc. 1143, 3 (2009). doi:10.1063/1.3169265

14. P. S. Ho, E. Zschech, D. Schmeisser, M. A. Meyer, R. Huebner, M. Hauschildt, L. J. Zhang, M. Gall and M. Kraatz, International J. Mater. Res. 101, 216 (2010).

15. C. Durkan and M. E. Welland, Ultramicroscopy 82, 125 (2000). doi:10.1016/S0304-3991(99)00133-3

16. A. S. Oates, J. Appl. Phys. 79, 163 (1996). doi:10.1063/ $\underline{1.360925}$

17. J. R. Lloyd and J. J. Clement, Thin Solid Films 262, 135 (1995). doi:10.1016/0040-6090(94)05806-7

18. J. Niehof, H. C. De Graaff, A. J. Mouthaan and J. F. Verwey, Solid-State Elect. 38, 1817 (1995). doi:10.1016/ $\underline{0038-1101(94) 00285-\mathrm{N}}$
19. R. G. Filippi, R. A. Wachnik, C.P. Eng, D. Chidambarrao, P.C. Wang, J. F. White, M. A. Korhonen, T. M. Shaw, R. Rosenberg and T. D. Sullivan, J. Appl. Phys. 91, 5787 (2002). doi:10.1063/1.1459616

20. K. L. Lee and C. K. Hu, J. Appl. Phys. 78, 4428 (1995). doi:10.1063/1.359851

21. C.K. Hu, B. Luther, F. B. Kaufman, J. Hummel, C. Uzoh and D. J. Pearson, Thin Solid Films 262, 84 (1995). doi:10.1016/0040-6090(94)05807-5

22. R. Rosenberg, D. C. Edelstein, C.K. Hu and K. P. Rodbell, Annu. Rev. Mater. Sci. 30, 229 (2000). doi:10.1146/ annurev.matsci.30.1.229

23. http://en.wikipedia.org/wiki/Palladium

24. B. K. Jones, Y. Z. Xu, T. C. Denton and P. Zobbi, Microelectron. Reliab. 35, 13 (1995). doi:10.1016/00262714(94)P1834-Y

25. R. Rosenberg, J. Appl. Phys. 42, 5671 (1971). doi:10. $\underline{1063 / 1.1659998}$

26. S. A. Lytle and A. S. Oates, J. Appl. Phys. 71, 174 (1992). doi: $10.1063 / 1.350733$

27. J. R. Lloyd and R. H. Koch, Appl. Phys. Lett. 52, 194 (1988).doi:10.1063/1.99517

28. F. O. Hadeed and C. Durkan, Appl. Phys. Lett. 91, 123120 (2007). doi:10.1063/1.2785982

29. M. L. Trouwborst, S. J. van der Molen and B. J. Van Wees, J. Appl. Phys. 99, 114316 (2006). doi:10.1063/1. 2203410

30. Annie T. Huang, K. N. Tu and Y.S. Lai, J. Appl. Phys. 100, 033512 (2006)

31. H. Zhang, G. Wang, G. S. Cargill III, J. Electron. Mat. 36, 117 (2007). doi:10.1007/s11664-006-0067-3

32. Z. X. Wang, H. L. Xu, Z. Y. Zhang, S. Wang, L. Ding, Q. S. Zeng, L. J. Yang, T. Pei, X. L. Wang, M. Gao and L.-M. Peng, Nano Lett. 10, 2024 (2010). doi:10.1021/nl100022u

33. Z. X. Wang, Z. Y. Zhang, H. L. Xu, L. Ding, S. Wang and L.M. Peng, Appl. Phys. Lett. 96, 173104 (2010). doi:10. $\underline{1063 / 1.3413959}$ 